\title{
Cruzando el puente entre la realidad y la imaginación: Terapia cognitiva basada en la inferencia para el TOC.
}

Crossing the bridge between reality and imagination: Inference Based Cognitive Therapi for Obsessive Compulsive Disorder

Fecha de recepción: 10/06/2014

Fecha de aceptación: 22/06/2015
Sara Llorens Aguilar

Psicóloga Generalista Sanitaria. Práctica Privada. Ángel Pozo

Psicólogo Especialista en Psicología Clínica. Práctica Privada.

\section{resumen/abstract:}

En este artículo se pretende exponer una revisión teórica de las líneas generales de la Terapia Basada en la Inferencia (TBI) para el TOC. Este tipo de intervención surge en función de algunas de las limitaciones que parecen presentar tanto los modelos cognitivo-conductuales como su aplicación práctica. La TBI no pretende sustituir a dichos modelos sino incidir en otros aspectos no considerados por los mismos. Así como los modelos cognitivo conductuales se han centrado en la modificación de las creencias disfuncionales y en la interpretación de los pensamientos intrusivos, junto con un fuerte componente de Exposición con Prevención de Respuesta (EPR), la TBI incide en el proceso de confusión inferencial que da lugar a la duda obsesiva inicial, la cual es considerada como un proceso de inferencia en sí misma. Se expondrán lo que desde el punto de vista de la TBI se consideran las limitaciones de los modelos clásicos y se hará referencia a los principios y fases de intervención de la TBI, finalizando con un ejemplo práctico del enfoque inferencial que ilustre como aplicar en la práctica clínica el modelo explicado.

This article pretends to introduce the Inference Based Therapy (IBT) for the Obsessive Compulsive Disorder. This new intervention appears due to some of the limitations that the cognitive appraisal models (CAM) present in the clinical practice. The IBT does not pretend to replace these models but to emphasize other clinical aspects not considered until now. While CAM are focused in modifying irrational beliefs domains and appraisals along with a strong component of Exposure with Prevention Response, the IBT underlines the inferential confusion process as the origin of the initial doubt, which is considered as an inference by itself. In the present article, we introduce: what would be the CAM limitations from the IBT point of view, the IBT principles and the different phases of the therapeutic process. We finish with a practical case to illustrate how to apply the model in the clinical practice.

\section{palabras clave/keywords:}

Trastorno Obsesivo Compulsivo, duda, confusión inferencial, Terapia Basada en la Inferencia, Modelos CognitivoConductuales

Obsessive Compulsive Disorder, doubt, inferential confusion, Inference Based Therapy, Cognitive-Appraisal Models

\section{Poniendo el TOC en perspectiva}

Decía H. Poincaré que la ciencia se construye con hechos de igual modo que una casa se hace con ladrillos, pero que un conjunto de hechos no es una ciencia, al igual que un conjunto de ladrillos no es una casa. Los "hechos" con los que se ha construido el edificio del tratamiento psicológico del TOC desde los tiempos de Meyer, parecen bien cimentados. Para 
ello no tiene el lector más que consultar los diversos metaanálisis (Abramowitz 1996, 1997, 1998; Steketee y Shapiro, 1993; Hiss, Foa y Kozak, 1994; Van Balkom, 1994) y estudios de resultado al efecto, las guías del NICE (2006) etc. No obstante, por lo que hace a la EPR, no fue hasta 1998 cuando se le asignó por parte del División 12 del APA (Clambless y cols, 1998) su estatus de tratamiento "bien establecido".

Para cualquier clínico de "a pié" que se dedique a trabajar con problemas de TOC, la EPR sigue siendo el tratamiento de elección, al margen de todos los ingredientes cognitivos que se le quieran añadir al mismo, aunque, como es sabido, en ocasiones serán estas intervenciones cognitivas las que tendrán preeminencia sobre la propia EPR, como en los enfoques sobre la responsabilidad de Salkovskis y su grupo $(1985,1989,1999,1999$ a) ,los trabajos derivados de la teoría cognitiva de Rachman $(1997,1998,2003)$, el trabajo sobre contaminación mental $(1994,2015)$ también de Rachman, las aportaciones de Wilhelm y Steketee (2006) e incluso las valientes propuestas de Grayson $(2003,2010)$ sobre tolerancia a la incertidumbre disfrazada de EPR radical, por citar sólo algunos.

Sin embargo, los clínicos de "a pie" también somos conscientes de las limitaciones que ofrece la EPR y hasta dónde se le puede pedir y ello no es sólo algo que leemos en la literatura científica, sino que lo palpamos en nuestra práctica clínica de diversos modos, desde la dificultades y rechazo que a veces crea la propia EPR (Steketee, 1993), las mejorías limitadas que se consiguen (Fisher y Wells, 2005), recaídas, casos de gran complejidad y comorbilidad que requieren de unas condiciones e intensidad que resultan difíciles de implementar, etc.

Por ello, parece evidente que el "edificio" de los Modelos Cognitivo-Conductuales (MCC) y la EPR presenta ciertas carencias o "deterioros", algunos de ellos quizá debidos al paso del tiempo, como puedan ser los detalles de aplicación de los procedimientos; pero también otros que parecer afectan a sus cimientos, como pueden ser las citadas limitaciones en los principios de la EPR o de las terapias cognitivas "clásicas" basadas en los modelos de interpretación de las intrusiones, como señalan acertadamente O'Connor y Robillard (1995 y 1999) en el artículo, digamos, fundacional de los enfoques MBI.

Pretendemos en este artículo exponer algunas de estas dificultades y presentar los rasgos esenciales de la Terapia Cognitiva Basada en la Inferencia para el TOC como un acercamiento cognitivo basado en principios diferentes, al objeto de mejorar los problemas que comentamos seguidamente. Pero, no tema el lector, la psicología clínica, para bien o para mal, no es una ciencia exacta y por ello las nuevas aportaciones habremos de entenderlas como elementos añadidos ("reformas", si se nos permite la expresión), la mayoría de las veces, como es el caso, compatibles con las propuestas anteriores, aunque bien es cierto que los enfoques MBI van un poco más allá. Los planteamientos y procedimientos del enfoque MBI, en ningún caso anularán los planteamientos anteriores (como le sucediera a Gottlob Frege con la famosa carta de B. Russell), sino que supondrán una manera de enriquecer los MCC, como estamos viendo ya en los trabajos de Van Niekerk $(2009,2014)$ O'Connor y $^{\prime}$ Clark (2005) Cualquiera que tenga cierta experiencia en TOC habrá reparado en las "paradojas" derivadas de su fenomenología y presentación clínica que, sin tener el efecto devastador a nivel epistemológico de las paradojas aludidas de la teoría de conjuntos, sí que lo 
tienen (y mucho en ocasiones) en la vida de las personas: lavarse durante horas para seguir sintiéndose contaminado; los criterios imposibles y arbitrarios derivados del "just right"; la potenciación de la duda patológica y el alejamiento de los sentidos cuando se intenta reducir al mínimo la incertidumbre; la creciente convicción de peligrosidad - maldad vinculada a las obsesiones sexuales; las comprobaciones en el ámbito del "hit and run" que no conducen más que a mayor duda; o los efectos de los intentos de supresión de las intrusiones, por citar sólo algunos..... Y así un largo etcétera de situaciones idiosincrásicas que asolan la vida de muchas personas.

\section{Limitaciones de los modelos actuales y de su aplicación.}

Sería sumamente interesante si pudiésemos preguntar a buen número de terapeutas del ramo, dónde y qué dificultades encuentran en su trabajo cotidiano con el TOC, lo que nos proporcionaría una información muy enriquecedora. Pero en este caso, tendremos que conformarnos con un análisis general de dichas dificultades o limitaciones. Si tuviésemos que subrayar algunas de las dificultades que presentan las intervenciones tradicionales en el TOC, diríamos, por lo que se refiere a la EPR, que raramente elimina todos los rituales (como decía un clínico: "como si hacer 4 horas de rituales en lugar de 6, fuese un avance significativo"), al margen de que entre el $25 \%$ y el $40 \%$ de los pacientes son incapaces de cumplir las pautas EPR. En algunos casos, la fuente directa de la ansiedad (suciedad, contaminación, incertidumbre, etc) en realidad no es un objeto fóbico para el paciente con TOC, porque éste no teme a los estímulos fóbicos del mismo modo que un fóbico a las arañas teme a las arañas y su foco se centra en los aspectos simbólicos de dichos estímulos más allá de sus propiedades físicas (O'Connor y Robillard, 1995). Esto es algo que observamos con claridad en las obsesiones de Contaminación Mental (Rachman, 1994, 2015) en sus diversas formas de contaminación derivada de violación física o psicológica, auto-contaminación, contaminación visual y "morphing". Remitimos al lector al excelente trabajo de Rachman (2015) al respecto.

Por otra parte existen una serie de dificultades que el terapeuta ha de atender en el desarrollo de las exposiciones (Gillihan y cols., 2012) como son la escasa intensidad de las mismas, el manejo inadecuado de la distracción y la reaseguración, la selección de formatos de exposición erróneos, la no atención a los rituales mentales, el no implicar a los familiares, la aplicación inadecuada de la prevención de respuesta, el no llevar a cabo la exposición en forma de "estilo de vida" (Abramowitz y cols., 2003) También las dificultades que se producen en la habituación de la ansiedad, la estimación inadecuada de la dificultad de las exposiciones o la presencia de niveles elevados de sensibilidad a la ansiedad (Pence y cols., 2010).

Por lo que se refiere a las terapias cognitivas vinculadas a los modelos clásicos de interpretación derivados de los Dominios de Creencias (OCCWG, 1997;Salkovskis, 1985, 1989, 1999; Rachman, 1997, 1998, 2003) existen algunas cuestiones que deben tenerse en cuenta a la hora de valorar su eficacia y planteamientos de base y que pueden ponerlas a prueba. Sin pretender hacer un listado exhaustivo, podemos citar las siguientes:

a) Algunos subtipos de TOC no muestran prácticamente mejoras con los MCC ni con la terapia cognitiva (por ejemplo,aquellos con ideación sobrevalorada, bajo insight u obsesiones de carácter egosintónico) (Kozak y Foa, 1994; Veale, 2002) 
b) En algunos cuadros clínicos, por ejemplo los de tipo "just right" resulta difícil encontrar creencias catastróficas asociadas.

c) Las terapias cognitivas actuales para el TOC (OCCWG, 1997) hacen hincapié y centran su objetivo de tratamiento en la interpretación disfuncional de los pensamientos intrusivos, dejando a un lado el análisis de las condiciones de aparición de la duda inicial y sus intrusiones asociadas, cuestión esta que parece ser determinante, como luego veremos, en buen número de casos (O’Connor, 2002)

d) No parece estar tan claro que los pensamientos intrusivos sean una experiencia universal tal como propone uno de los principios de los modelos CAM (es decir, la idea de que todos tenemos pensamientos intrusivos, pero la diferencia radica en su interpretación, frecuencia, etc. (No obstante, tenga en cuenta el lector que los términos "modelos CAM" y "MCC" no son enteramente superponibles). En este aspecto, la "relevancia" del contexto en el que surge la actividad intrusiva podría ser un factor distintivo más relevante entre PI "normales", (que surgirían dentro de un contexto apropiado) y los clínicos (que estarían alimentados por la narrativa ficticia interna propia del TOC) (Clark y O’Connor, 2005)

e) Las intervenciones cognitivas derivadas de los MCC, raramente se van a emplear por sí solas y no parecen aportar mejoras sustanciales al proceso terapéutico, en el que la EPR sigue siendo el ingrediente principal. (Fama y Wilhelm, 2005).

f) Pese a que las intervenciones derivadas de los MCC son efectivas, no lo son más que la EPR a la hora de reducir síntomas TOC o provocar cambios en los Dominios de Creencias. Es más, incluso antes de la propia intervención, la mitad de cualquier muestra con TOC no va a ofrecer puntuaciones elevadas en las escalas clínicas que evalúan los distintos Dominios de Creencias (por ej, el Obsessive Beliefs Questionnaire). Asimismo, muchos de los Dominios de Creencias derivados de los MCC no son específicos del TOC, sino que son compartidos por otros problemas de ansiedad (Tolin, 2006).

g) No parece haber evidencia de que la presencia de interpretaciones disfuncionales de las intrusiones sea un factor relevante en el inicio del TOC, ya que puede ser también una consecuencia del mismo (Abramowitz, 2007; Mancini, 2002).

h) Los modelos CAM se centran en la interpretación de la intrusión y en la cadena de consecuencias derivadas de la misma, probabilidades, responsabilidad personal, etcétera; todos ellos factores de suma importancia, pero pasan por alto el componente clave de la duda inicial y los procesos de Confusión Inferencial vinculados a la misma, tal como propone el Modelo Basado en la Inferencia (Inference Based Approach, en inglés) que veremos a continuación (Aardema y cols, 2005a y 2005b)

\section{Un punto de vista diferente: El Modelo Basado en la Inferencia}

En la actualidad se considera que el Trastorno Obsesivo Compulsivo afecta alrededor de un $2.5 \%$ de la población. A lo largo del tiempo, muchos han sido los intentos de tratamiento psicológico pero pocos de ellos han llegado a obtener resultados exitosos (de forma significativa). La EPR es la estrategia terapéutica que ha demostrado mayor eficacia hasta 
el momento (Chambless y cols, 1998) pero parece claro que un porcentaje relativamente elevado de la población clínica, o bien la rechaza o bien no obtiene resultados enteramente positivos(Julien, O’Connor y Aardema, 2006) Este hecho ha dado lugar a diversas investigaciones cuyo objetivo es buscar terapias alternativas para poder ayudar a esta parte de la población clínica que actualmente no encuentra una salida viable a su situación. Una de estas líneas de investigación es el Modelo Basado en la Inferencia que surgió hace algunos años (1995) en Canadá a manos de Kieron O’Connor y su grupo de trabajo. Se trata de una terapia de tipo cognitivo cuya principal hipótesis se basa en la existencia de un razonamiento inductivo erróneo como principal factor de origen y mantenimiento del TOC. Este razonamiento erróneo, llamado Confusión Inferencial (CI) , sería la clave del mantenimiento del trastorno.

Para que nos entendamos mejor, un razonamiento inductivo correcto sigue este proceso: 1) forrmulación de una hipótesis, 2) observación, 3) comprobación de si los hechos validan o no mi hipótesis, y 4) conclusiones. En cambio, el razonamiento inductivo en el TOC según el MBI seguiría éste otro PROCESO: 1) formulo una hipótesis y la doy por válida 2) busco pruebas que confirmen la validez que arbitrariamente le he dado a mi hipótesis.

Un ejemplo de razonamiento inductivo correcto sería el siguiente

1. Formulación de una hipótesis: Puede que tenga las manos sucias o contaminadas.

2. Observación: Las miro para ver si hay signos de suciedad o contaminación.

3. Comprobación de si los hechos validan o no mi hipótesis: No encuentro manchas ni rastros que indiquen que mis manos pueden estar sucias o contaminadas.

4. Conclusiones: Tengo las manos limpias.

Según el MBI, un ejemplo de razonamiento inductivo en un caso de TOC sería éste:

1. Formulo una hipótesis y la doy por válida: Tengo las manos sucias

2. Busco pruebas que confirmen la validez que arbitrariamente le he dado a mi hipótesis: aunque no pueda ver manchas ni huelan mal,creo que están sucias. Los gérmenes existen pero no los podemos ver, por lo tanto podrían estar contaminadas y no poder apreciarlo visualmente. Que no pueda verlos, no significa que no puedan estar ahí.

El MBI fue diseñado especialmente para los casos de TOC con bajo o nulo insight, es decir, para aquellas personas que no son tan conscientes de la irracionalidad de sus obsesiones, al contrario de lo que sucede con la gran mayoría de personas que padecen este trastorno, cuyo nivel de insight suele ser elevado(Kozak y Foa, 1994). Estudios a lo largo de los últimos años (O'Connor y Robillard,1995; Aardema et al. 2005a; O'Connor, Aardema, Bouthillier, Fournier,Guay, Robillard,Pélissier y Landry, 2005)han confirmado que el MBI es eficaz en todos los casos de TOC y no sólo para aquellos con bajos niveles de insight, con lo que se presenta en el ámbito clínico como una nueva e interesante alternativa de tratamiento para este trastorno.

El MBI de O’Connor presenta varias e importantes diferencias con los MCC que actualmente inspiran las intervenciones cognitivas para el tratamiento del TOC (Salkovskis, 
1985,1989; Purdon y Clark, 1999;Wells, 1997;Rachman 1997,1998). El MBI pretende superar las dificultades de dichos modelos, que el lector puede repasar en el metaanálisis realizado por Julien, O'Connor y Aardema (2006) en el que queda patente que las terapias actuales para el TOC presentan interrogantes que dejan clara la necesidad de seguir investigando en el área. Veamos una breve introducción de estos puntos, que obviamente definirán las diferencias en los objetivos y focos de intervención (O’Connor, Aardema, Pélissier, 2005):

1. En primer lugar, el MBI plantea que el TOC no consiste en un problema de percepción sino de imaginación. No afirma que las interpretaciones erróneas de la obsesión no influyan en el origen y mantenimiento del problema, sino que éstas no son el factor principal que mantiene el trastorno, tal y como afirma los MCC. Por tanto, el problema no solo reside en cómo el paciente interpreta los pensamientos intrusivos sino en el razonamiento inductivo derivado de los procesos de imaginación que genera el propio individuo a partir de dichos pensamientos. Este razonamiento inductivo incorrecto suele ser específico al ámbito del TOC, lo que significa que el individuo razona correctamente en el resto de áreas de su vida que no se ven afectadas por los procesos obsesivos. Es decir, un paciente con TOC puede no fiarse de que sus manos están limpias aunque vea que esto es así o puede no fiarse del "clac" que la llave hace al cerrar la puerta de su casa porque sus obsesiones se relacionan con estas temáticas, sin embargo sí se fiará del color del muñequito verde del semáforo que le indica que como peatón puede cruzar o de que su email no contiene ninguna falta de ortografía tras revisarlo antes del envío. La desconfianza en sus cinco sentidos solo se limita a su área(s) TOC.

El razonamiento inductivo erróneo o confusión inferencial se basa en dos puntos clave:

a) Desconfianza de los propios sentidos. La persona no distingue lo real de lo imaginado (al menos en el ámbito del TOC) porque no se fía del criterio universal que utilizamos los seres humanos para hacer dicha distinción: nuestros cinco sentidos.

b) La asignación de una mayor credibilidad a una posibilidad ficticia remota que a la realidad. Es esencial tener en cuenta que cuando hablamos de ficticio, no hacemos referencia a una imposibilidad física sino que nos estamos refiriendo a algo que no se está dando o que no es relevante en el aquí y ahora, en la realidad que está viviendo el individuo.

2. En segundo lugar, el MBI se distancia del modelo fóbico en el que se basan los MCC para el tratamiento del TOC. Ello se debe a que, como se ha dicho anteriormente, no siempre existe un paralelismo entre la obsesión y el estímulo fóbico o sus propiedades objetivas, ya que afirma que la naturaleza (simbólica en ocasiones) del fenómeno que representa la obsesión es bien distinta de las características topográficas del estímulo fóbico.

Por ejemplo, un radiofísico que padece un TOC de contaminación y que trabaja en contacto con pacientes oncológicos, actúa como si la enfermedad (el cáncer) fuera contagiosa cuando sabe con certeza que no lo es. Es aquí cuando el estímulo fóbico ha dejado de serlo desde un punto de vista objetivo pero sigue siéndolo subjetivamente para la persona.

3. En tercer lugar, el MBI cuestiona la idea de que los pensamientos intrusivos sean fenómenos o experiencias universales, tal como afirman los MCC. El MBI afirma que hay una 
diferencia importante en lo que al contexto se refiere. Si bien es cierto que la población general suele experimentar pensamientos intrusivos, estos surgen en contextos apropiados, no sucediendo lo mismo con los pensamientos intrusivos de la población clínica, que aparecerían en contextos anómalos.

Siguiendo el ejemplo anterior, la preocupación de nuestro radiofísico aparece en un contexto inapropiado, ya que evita el contacto con pacientes con cáncer por miedo al contagio, aun sabiendo que esta enfermedad no es contagiosa (contexto inapropiado). Es decir, el qué tiene sentido porque es cierto que el cáncer es una enfermedad mortal pero el cómo y el cuándo convierten al miedo en irracional, ya que en el aquí y ahora de nuestro paciente no es posible un contagio.

El tipo de contexto en el que surgen las intrusiones sería una variable esencial, lo que nos lleva a plantearnos la posibilidad de que en lugar de representar un continuo, los pensamientos intrusivos y las obsesiones sean en realidad dos fenómenos diferentes siendo los primeros los que se suelen presentar en la población normal, mientras que las segundas serían propias de la población clínica.

4. Por otra parte, el MBI afirma que la intervención ha de centrarse en la duda obsesiva (inferencia primaria) y no tanto en las consecuencias/interpretaciones (inferencias secundarias). Esto adquiere sentido cuando uno entiende que si la duda obsesiva deriva de un proceso imaginario (sin base en el aquí y ahora), las consecuencias, por tanto, también lo son. Por ello, el objetivo del terapeuta habrá de desplazarse de las consecuencias catastróficas imaginarias y de su probabilidad o alcance a la duda obsesiva que las precede, la cual ya es en sí misma una inferencia errónea. De hecho, diversos estudios en la TBI han comprobado que trabajando y disminuyendo la inferencia primaria a través de la modificación del razonamiento inductivo que lleva a la misma, disminuye de forma indirecta la convicción en la inferencia secundaria.

\section{Estímulo externo o interno}

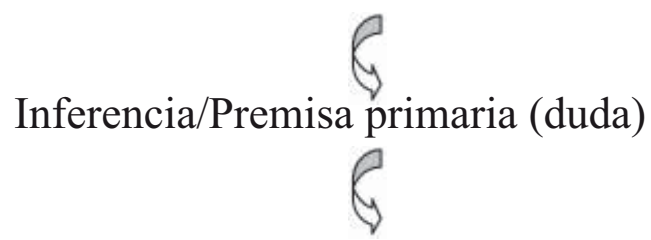

Inferencias/premisas Secundarias (consecuencias negativas de la primaria)<smiles>c1ccccc1</smiles>

Malestar generado por las inferencias secundarias

Conductas para mitigar el malestar y/o la amenaza (compulsiones)

Figura 1. Modelo Inferencial para el TOC (O’Connor, 2002) 
Siguiendo con nuestro ejemplo, la inferencia primaria o duda obsesiva del paciente sería la siguiente: "¿Y si esta paciente pudiera haberme transmitido el cáncer de una forma desconocida hasta el momento?" Y como consecuencias derivadas de la duda obsesiva o inferencias secundarias tendríamos: "Entonces yo podría contagiárselo a otras personas. Podría llegar a contagiárselo a mi familia y sería culpable de que mi mujer y mis hijos padecieran cáncer...."

5. Otra diferencia crucial radica en la importancia que se le asigna al contenido de los pensamientos intrusivos. El MBI sugiere que el contenido de las dudas obsesivas es temático y significativamente personal, vinculado a factores de vulnerabilidad como afectividad negativa elevada, aprehensión ansiosa, autoevaluaciones ambivalentes, narrativas inductivas idiósincrásicas o creencias metacognitivas disfuncionales (Clark y O 'Connor, 2005) a diferencia de los MCC que sostienen que el contenido de las obsesiones es azaroso; o vinculado a procesos genéricos de estrés. El MBI sugiere que el "tema" o contenido está relacionado con un self ficticio con connotaciones negativas; ; es decir, la persona, al menos en el ámbito del TOC, se comporta en base a quién podría ser o llegar a ser en lugar de cómo quién es en realidad (Aardema y O'Connor, 2007). Este aspecto da lugar en muchas ocasiones a un tipo de proceso de fusión (que los autores denominan Fusión de Pensamiento - Pensamiento), a añadir a la ya conocida lista de las distintas variedades de Fusión de Pensamiento - Acción, por el que pensar en la posibilidad de tener determinado pensamiento intrusivo (que es lo que suele ocurrir habitualmente) se equipara con haberlo tenido en realidad. Por ejemplo, un joven de 23 años piensa " ¿y si llegara a tener una fantasía sexual con un hombre?” En este caso el paciente confunde la posibilidad de tener un pensamiento homosexual con el hecho de tenerlo y lo que esto representaría para él (confirmar su homosexualidad).

Otro ejemplo que ilustra bien este sesgo es el de una madre que viendo a su hijo se plantea la posibilidad de llegar a pensar en hacerle daño y que confunde dicha posibilidad con haber tenido el pensamiento.

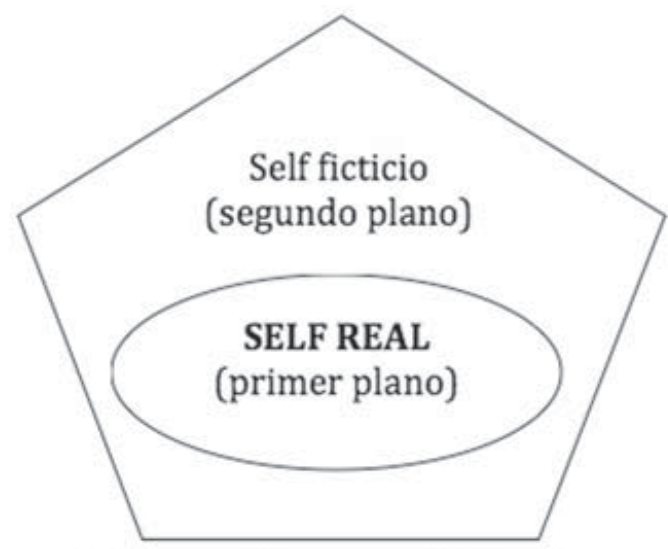

1. Percecpión normal del self

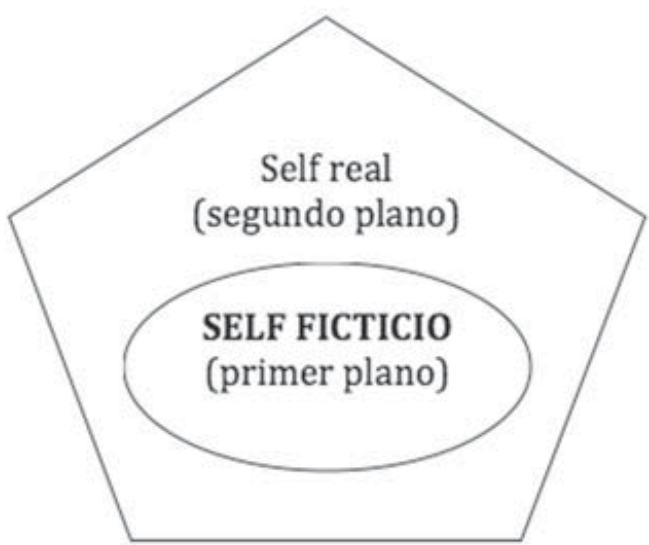

2. Percepción obsesiva del self

Figura 2. Relación normal y obsesiva del self real y self ficticio(Aardema F. y O'Connor, 2007) 
A continuación, en la Tabla 1, se resumen las principales diferencias entre los MCC y el MBI

\begin{tabular}{|c|c|c|}
\hline CARACTERÍSTICA & $\begin{array}{l}\text { MODELO COGNITIVO- } \\
\text { CONDUCTUAL }\end{array}$ & $\begin{array}{c}\text { MODELO } \\
\text { INFERENCIAL }\end{array}$ \\
\hline $\begin{array}{c}\text { Intrusión/Primera infe- } \\
\text { rencia }\end{array}$ & $\begin{array}{l}\text { - Universal y derivada de } \\
\text { procesos genéricos de estrés. } \\
\text {-El contenido no es impor- } \\
\text { tante }\end{array}$ & $\begin{array}{l}\text { - No es universal y está vin- } \\
\text { culada al contexto y al self. } \\
\text {-Importancia del contenido y } \\
\text { contexto }\end{array}$ \\
\hline $\begin{array}{l}\text { Factor desencadenante } \\
\text { del problema }\end{array}$ & $\begin{array}{l}\text { Una malinterpretación de la } \\
\text { intrusión conduciría a la ob- } \\
\text { sesión. }\end{array}$ & $\begin{array}{l}\text { Un razonamiento inductivo } \\
\text { erróneo conduciría a la obse- } \\
\text { sión (narrativa ficticia). }\end{array}$ \\
\hline $\begin{array}{c}\text { Factores mantenedores } \\
\text { del problema }\end{array}$ & $\begin{array}{l}\text { Cualquier tipo de conducta } \\
\text { compulsiva (manifiesta y en- } \\
\text { cubierta) así como conductas } \\
\text { de neutralización. }\end{array}$ & $\begin{array}{l}\text { Errores de razonamiento que } \\
\text { originan y mantienen la na- } \\
\text { rrativa ficticia: asociaciones } \\
\text { irrelevantes... }\end{array}$ \\
\hline Origen del problema & $\begin{array}{l}\text { Dominios de creencias disfun- } \\
\text { cionales }\end{array}$ & $\begin{array}{l}\text { Una percepción negativa } \\
\text { errónea del propio self }\end{array}$ \\
\hline Paciente prototipo & $\begin{array}{l}\text { Cualquier tipo de TOC, espe- } \\
\text { cialmente aquellos con cierto } \\
\text { grado de insight. }\end{array}$ & $\begin{array}{l}\text { Cualquier tipo de TOC, espe- } \\
\text { cialmente aquellos con bajo } \\
\text { insight. }\end{array}$ \\
\hline $\begin{array}{c}\text { Objetivo del tratamiento } \\
\text { y tipo de técnicas que se } \\
\text { emplean. }\end{array}$ & $\begin{array}{l}\text { Modificar la interpretación sin } \\
\text { tener en cuenta el contenido. } \\
\text { Se hace a través de la EPR y } \\
\text { modificación de dominios de } \\
\text { creencias disfuncionales. }\end{array}$ & $\begin{array}{l}\text { Modificar la narrativa ficticia } \\
\text { a través de la Terapia Infe- } \\
\text { rencial, que consiste en una } \\
\text { serie de pasos de tipo cog- } \\
\text { nitivo dirigidos a mejorar el } \\
\text { razonamiento inductivo del } \\
\text { paciente. }\end{array}$ \\
\hline
\end{tabular}
Carácter del problema
De tipo perceptivo
De tipo imaginativo

Tabla 1. Diferencias entre los modelos Cognitivo-Conductual e Inferencial.

\section{Formas nuevas de trabajar: Terapia basada en la Inferencia}

Considerando estos planteamientos teóricos el proceso terapéutico irá vinculado a los mismos. Mientras que el objetivo de la TCC sería modificar las interpretaciones disfuncionales que el paciente hace de sus obsesiones, el objetivo de la Terapia Basada en la Inferencia (TBI) sería conseguir que el paciente distinguiera claramente entre lo que es (realidad), lo 
que podría ser (probabilidad remota) y la ficción. Para ello, la TBI va a centrarse en que la historia o narrativa del TOC creada por el paciente vaya perdiendo credibilidad y siendo sustituida por otra historia o narrativa alternativa al TOC basada en los sentidos, en el aquí $y$ ahora. El proceso terapéutico se divide en nueve fases. Aunque aquí no nos paremos a describir el contenido de los mismas, la TBI ha diseñado una serie de ejercicios que corresponden a las diferentes etapas que constituyen la terapia y que el lector puede consultar en los dos manuales de referencia que se citan en la bibliografía (O'Connor, K.P y cols, 2005; $\mathrm{O}^{\prime}$ Connor, K.P y Aardema, F., 2012) Estos ejercicios sirven para que el paciente practique en su día a día lo que se le va explicando y trabajando a lo largo de las sesiones.

Vamos con las fases que componen la terapia (O’Connor, K.P y Aardema, F.,2012):

1. Evaluación:Además de la evaluación conductual al uso, en esta fase evaluaremos el nivel de capacidad de resistencia a las compulsiones, los estados de ánimo y emociones asociadas a la duda obsesiva (no siempre es ansiedad), la probabilidad de la Inferencia Primaria (IP) de 0 a 100,la fuerza de la Inferencia Secundaria (IS) de 0 a 100 y por último, la convicción (0-100) a la hora de necesitar realizar compulsiones tanto en situaciones TOC como no TOC.

El Inferential Confusion Questionnaire (Aardema, F., O’Connor, K. P., Emmelkamp, P. M., Marchand, A., y Todorov, C., 2005b) diseñado por el mismo grupo de trabajo, es la herramienta principal para conocer el nivel de CI (Confusión Inferencial) que presenta el paciente.

2. Dudas normales versus dudas obsesivas: En esta fase explicamos al paciente que existen diferencias esenciales entre las dudas cotidianas y las dudas obsesivas. Estas diferencias nos ayudan a distinguir cuándo hablamos de un tipo de duda u otro y consecuentemente nos indican cómo actuar según la situación en la que nos encontremos. Explicaremos al paciente dichas diferencias y las extrapolaremos a su caso con el objetivo de que aprenda a distinguir cuando su duda es obsesiva y por qué lo es.

3. La lógica del TOC: explicaremos al paciente el razonamiento inductivo erróneo que le ha llevado a construir su narración TOC y a asignarle credibilidad. Trabajaremos en diferenciar un razonamiento inductivo pertinente al aqui y ahora frente a otro anómalo (más allá del aquí y ahora) y mediante el empleo de una serie de ejemplos relevantes al caso se guiará socráticamente al paciente hacia la construcción de una narrativa diferente basada en el aquí y ahora. Este razonamiento se traduce en varios tipos de argumentos o mecanismos que emplea el TOC para perpetuarse (como si se tratase de autoreplicadores meméticos) que también explicaremos al paciente para que los identifique en su propia área obsesiva.

4. La duda obsesiva es $100 \%$ irrelevante para el aquí y ahora: En esta fase explicamos al paciente que lo importante no es qué justifica la duda obsesiva, (no es relevante que la probabilidad de contagiarse de x sea extremadamente pequeña)sino el contexto, es decir, el cómo y cuándo (el cáncer no es una enfermedad contagiosa, por lo tanto la posibilidad de contagio a través de los pacientes es irrelevante para el aquí y ahora). Si los argumentos no están basados en su aquí y ahora, entonces no son relevantes porque no reflejan la 
realidad que vive el individuo y desde ese momento pasan a formar parte del ámbito de la imaginación.

5. La narración: explicamos la gran influencia que nuestro discurso interno tiene sobre nosotros y nuestras creencias. Sentimos y actuamos en base a lo que nos vamos diciendo. Para que la duda obsesiva pierda credibilidad, habrá que crear nuevas historias o narrativas más realistas (ancladas al aquí y ahora) que sustituyan la actual historia TOC.

6. Punto de cruce: En esta fase enseñamos al paciente a identificar el punto de cruce entre realidad y ficción, es decir, el momento de su discurso interno en el que deja de confiar en sus cinco sentidos, en su aquí y ahora, para pasar al mundo de la ficción en el que cualquier cosa es posible por improbable, anómala o extraña que resulte al análisis racional y a los cinco sentidos.

7. Herramientas del TOC: El trastorno se vale diferentes herramientas o estrategias de razonamiento para dar credibilidad a la narración TOC que se ha ido creando y hacer que parezca real (recuerde el lector que desde el punto de vista epistemológico estamos considerando este proceso desde una analogía memética). Todas estas herramientas son argumentos que van más allá de los cinco sentidos y es nuestro trabajo enseñárselas al paciente para que pueda identificarlas en su propia narrativa. Las herramientas de razonamiento del TOC se clasifican de la siguiente forma:

- Errores de categoría: Se dan cuando se confunde dos categorías de información o de objetos, estableciendo entre ellos una relación que no existe.

- Situaciones aparentemente comparables: consiste en confundir dos situaciones diferentes que están separadas en tiempo y espacio.

- Uso selectivo de hechos sacados de contexto: hechos abstractos se aplican de forma inapropiada a contextos personales específicos.

- Secuencias puramente ficticias: Inventar historias convincentes y vivirlas.

- Inferencia inversa: realizar inferencias que preceden la observación o la realidad en lugar de seguirla.

- Desconfianza de la percepción normal: Desconfiar de los propios sentidos a favor de una realidad "más profunda".

8. Esquemas de vulnerabilidad (self themes): según el MBI la vulnerabilidad al TOC está relacionada con una autoimagen negativa basada en un self ficticio, al que se le da más credibilidad que a la identidad o self real. En esta fase se exploran esos esquemas en los que el paciente es propenso a elaborar una autoimagen negativa que no corresponde con la real y le ayudaremos a cambiar esa autoimagen por una más realista.

9. Tolerando el vacío: En la última fase se trabajará con el paciente la sensación de vacío (de tipo "debería hacer algo más" o " no es suficiente”) derivada de la desaparición progresiva de sus rituales y compulsiones (recuerde el lector que en la TBI no se ha hecho un trabajo específico de EPR, aun no es incompatible con la misma, sino que éste se obtiene de manera natural a través de giro narrativo que se desarrolla a lo largo de todo el proceso 
terapéutico). Esa sensación es la que deja la reducción gradual de compulsiones. Cuanto más tiempo pase y más se habitúe a no realizarlas, es decir, a llevar un estilo de vida coherente con la narrativa anclada en el aquí y ahora, dicha sensación irá desapareciendo.

\section{El caso de Elena}

Para ilustrar de forma práctica todo lo dicho anteriormente, a continuación relatamos el caso de una paciente con la que se trabajó en base al modelo.

Elena, una profesora de 35 años casada y madre de un niño. Empezó con obsesiones de agresividad hace 7 años. Solía tener imágenes de sí misma dañando a desconocidos con algún tipo de utensilio (cuchillo, cuerdas, objetos pesados...) o empujando por el balcón a varios miembros de su familia. Estos pensamientos en forma de imágenes se acentuaron tras dar a luz a su hijo Juan. Cuando Juan cumplió 2 años, Elena pasó por un periodo bastante estresante, con problemas en el trabajo y en su matrimonio, y fue entonces cuando las obsesiones volvieron con más fuerza que nunca. Dejó pasar un tiempo para ver si éstas desaparecían y al ver que esto no sucedía decidió buscar ayuda profesional.

Narración TOC de Elena "Si pienso en hacerle daño a Juan (inferencia primaria o duda), entonces significa que de forma oculta no le quiero y que realmente podría hacerle daño(inferencia secundaria o consecuencias derivadas de la duda). Si te das cuenta, tiene sentido. Fíjate en las noticias, solo hace falta encender la TV o abrir un periódico para darse cuenta de que no es ninguna tontería. Esas cosas ocurren. El otro día mismo comentaban que una madre había envenenado a su niña de 6 años y la había matado iqué horror! ¿Quién te dice a ti que no podría ser yo en uno de esos momentos en los que me bloqueo? Hay gente muy normal que "pierde la cabeza" en un momento dado. Cuando se piensa algo es por una razón, las ideas como éstas no surgen de la nada. Además, hace 10 años me pelee con mi mejor amiga y llegué a perder el control, llegué a abofeteara y tirarle de los pelos, lo que quiere decir que tengo cierta tendencia a la agresividad, a perder el control. Si eso es así, mis hijos están en peligro cuando estoy a solas con ellos. Si tengo facilidad para perder los nervios, eso quiere decir que soy una persona agresiva. A veces, en momentos de mucho bloqueo me he llegado a plantear ¿Y si les he pegado o les he hecho daño y no me acuerdo? No recuerdo haberles gritado o tocado y de hecho les miro y no tienen marcas ni lloran ni están tristes pero... es como si no me fiara ¿sabes? Como si yo supiera que no ha pasado pero no terminara de creérmelo"

Teniendo en cuenta la anterior clasificación de las herramientas del TOC, en esta narrativa observaríamos las siguientes:

Situaciones aparentemente comparables: El otro día mismo comentaban que una madre había envenenado a su niña de 6 años y la había matado iqué horror! ¿Quién te dice a ti que no podría ser yo en uno de esos momentos en los que me bloqueo?

Uso selectivo de hechos sacados de contexto: Hace 10 años me peleé con mi mejor amiga y llegué a perder el control, llegué a abofeteara y tirarle de los pelos, lo que quiere decir que tengo cierta tendencia a la agresividad, a perder el control. Si eso es así, mi hijo está en peligro cuando estoy a solas con él. 
Errores de categoría: Si tengo facilidad para perder los nervios, eso quiere decir que soy una persona agresiva

Desconfianza de los propios sentidos: $A$ veces, en momentos de mucho bloqueo me he llegado a plantear ¿Y si les he pegado o les he hecho daño y no me acuerdo? No recuerdo haberles gritado o tocado y de hecho les miro y no tienen marcas ni lloran ni están tristes pero... es como si no me fiara ¿sabes? Como si yo supiera que no ha pasado pero no terminara de creérmelo"

Aunque no vamos a entrar en clasificaciones sobre los diferentes tipos de mecanismos argumentales que utiliza, sí podemos estar de acuerdo en que ninguno de ellos se basa en el aquí y ahora de Elena, no se basan en hechos que reflejen la realidad de la paciente. Observamos que en un principio la narración parece bastante racional, resulta creíble y tiene sentido pero lo pierde cuando ubicamos la historia, ya que no tiene nada que ver con el contexto de la persona que la narra. Aquí observamos lo que mencionamos anteriormente: el problema no está en el qué sino en el cómo y cuándo, que son igual o incluso más importantes que el contenido en sí mismo.

La última línea ya nos está dando pistas de cuáles son los self themes de Elena, el self ficticio con el que erróneamente se identifica: "Soy el tipo de persona que podría perder el control", "Soy el tipo de persona capaz de agredir a otros".

Ahora nos vamos a unas sesiones más tarde y revisamos la narrativa alternativa que se ha ido elaborando a lo largo del proceso terapéutico. Esta historia se basa en hechos, en argumentos basados en los cinco sentidos y en el aquí y ahora de la paciente.

"Es cierto que existen madres que hacen daño a sus hijos, desgraciadamente es un hecho pero eso no tiene nada que ver conmigo. Yo quiero a Juan y nunca le he hecho daño, es más, siempre le cuido cuando está enfermo, le llevo al médico, le compro las medicinas que necesita, pido días en el instituto para quedarme en casa y cuidarle, me angustio cuando no sé qué le pasa y he llegado a estar más de dos dias sin comer cuando él ha estado malo. Si quisiera hacerle daño, no actuaría de este modo. Nunca he intentado agredirle de ninguna forma, es más, estoy constantemente vigilándolo para que no se caiga o se dé contra algo. No soy una persona agresiva porque hace diez años tuviera una pelea fuerte con una amiga. Son cosas que pueden pasar y una acción no define a una persona. Es más, cuando algo no me gusta, siempre intento hablarlo, dialogar y llegar a un acuerdo. No suelo chillar y nunca he vuelto a pegar a nadie. Me puedo sentir furiosa alguna vez pero tener esas emociones no significa ser agresiva. Tengo derecho a sentirme triste, enfadada o como todo el mundo y eso no me convierte en alguien peligroso porque entonces todos lo seríamos. A todos nos puede dar un ataque de locura y perder el control, yo no tengo más probabilidades que los demás. También es posible que pueda tener un accidente con la moto y no por eso dejo de utilizarla. O también es posible que me olvide los fuegos encendidos y no por eso dejo de usar mi cocina. No puedo basar mis actuaciones en posibilidades remotas jeso sí es una locura, no viviría!"

Como podemos observar, la narrativa alternativa se limita a los hechos que acontecen en la vida de la paciente, a su aquí y ahora. De forma que en esta historia no solo el "qué" es co- 
rrecto, sino que también lo son el "cómo" y el "cuándo". Refleja claramente como distingue entre posibilidad, probabilidad y realidad, principal objetivo de la TBI.

\section{Hacia dónde nos encaminamos}

En la actualidad, el modelo centra su atención en profundizar en el análisis de los esquemas de vulnerabilidad (Aardema, Moulding, Randomsky, Doron, Allambi y Souki, 2013; Melli, Aardema y Moulding, 2015) yen cómo éstos se relacionan con el origen y mantenimiento del trastorno. En lo que respecta a la aplicación de la TBI a otras áreas, trastornos como la Anorexia Nerviosa (AN), Bulimia Nerviosa (BN) o Trastorno por Acumulación Compulsiva (TAC) son objeto de estudio actual. Aún es pronto para afirmar nada pero parece ser que se han obtenido resultados significativos en el tratamiento de la BN, no siendo tan esperanzadores en la AN.(Purcell Lalonde y O'Connor et al. 2015)

Como sabe el lector, el desarrollo de cualquier empresa científica, como lo es la psicología clínica, es un proceso que nunca acaba. Por tanto, hemos de estar abiertos a conocer e investigar todas aquellas alternativas que puedan proporcionarnos aportaciones significativas porque solo así lograremos avanzar. No se trata de aceptar a ciegas toda la información que nos llega sin criterio alguno, sino de estar abiertos a nuevas posibilidades, a nuevos puntos de vista que tras su validación empírica puedan abrirnos las puertas que a día de hoy permanecen cerradas para algunos pacientes. En definitiva, que puedan hacer que esas deficiencias del edificio del tratamiento psicológico del TOC puedan ir corrigiéndose, de modo que nuestro querido Poincaré, aunque no era psicólogo, quedase satisfecho con las reformas.

\section{Referencias}

Aardema, F., Emmelkamp, PMG. \& O'Connor, K. (2005a). Inferential confusion, cognitive change, and treatment outcome in obsessive-compulsive disorder. Clinical Psychology and Psychotherapy ,12, 337-345.

Aardema, F., O'Connor, K., Emmelkamp, P.M.G, Marchand A. \& Todorov C. (2005b). Inferential confusion in obsessive-compulsive disorder: the inferential confusion questionnaire. Behaviour Research and Therapy, 44, 293-308.

Aardema, F., Kleijer, T. M. R., Trihey, M., 0 'Connor, K., \& Emmelkamp, P. (2006a). Inference processes, schizotypal thinking and obsessivecompulsive disorder. Psychological Reports, 99, 213-220

Aardema, F. \& O'Connor, K. (2007) The menace within: obsessions and the self. Journal of cognitive psychotherapy: an international quarterly, 21 (3), 182-197

Aardema, F., Moulding, R., Randomsky, A.,S., Doron, G., Allambi, J. and Souki, E (2013) Fear of self and obsessionality: Development and validation of the Fear of Self Questionnaire. Journal of Obseessive-Compulsive and Related Disorders, 2, 306-315

Abramowitz, J.S. (1996). Variants of exposure and response prevention in the treatment of obsessive- compulsive disorder: a meta-analyses. Behavior Therapy, 27, 583-600.

Abramowitz, J.S. (1997). Effectiveness of psychological and pharmacological treatments for obsessive-compulsive disorder: A quantitative review. Journal of Consulting and Clinical Psychology, 65, 44-52.

Abramowitz, J.S. (1998). Does cognitive behavioral therapy cure obsessive-compulsive disorder? A meta-analytic evaluation of clinical significance. Behavior Therapy, 29, 339-355.

Abramowitz, J.S., Franklin, M.E. \& Cahill, S. P. (2003). Approaches to common obstacles in the Exposure-Based treatment of ObsessiveCompulsive disorder. Cognitive and Behavioural Practice, 10, 14 - 22. 
Abramowitz, J.S, Nelson C.A., Rygwall R. \& Khandker M. (2007). The cognitive mediation of obsessive-compulsive symptoms: a longitudinal study. Journal of Anxiety Disorders , 21, 91-104.

Chambless, D. L., Baker, M. J., Baucom, D. H., Beutler, L. E., Calhoum, K. S., Crits-Christoph, P., Daiuto, A., DeRubeis, R., Detweiler, J., Haaga, D. A. F., Johnson, S. B., McCurry, S., Mueser, K. T., Pope, K. S., Sanderson, W. C., Shoham, V., Stickle, T., Williams, D. A. \&Woody, S. R. (1998). Update on Empirically Validated Therapies II. The Clinical Psychologist, 51, 3-15.

Clark, D.A \& O'Connor, K. (2005). Thinking is believing. Ego-Dystonic Intrusive Thoughts in Obsessive-Compulsive Disorder. In: Clark, D. A. Intrusive Thoughts in Clinical Disorders. Theory, Research and Treatment. Guilford.

Clark, D. A. \& Shelley, R.(2005) Unwanted Intrusive Thoughts in Nonclinical Individuals: Implications for Clinical Disorders. In: Clark, D. A. Intrusive Thoughts in Clinical Disorders. Theory, Research and Treatment. Guilford.

Fama, J. M. \& Wilhelm, S. (2005) Formal cognitive Therapy: A useful ingredient in the treatment of OCD. In: Abramowitz, J. S; Houts, A. C (eds.). Handbook of OCD: Concepts and controversies in Obsessive Compulsive Disorder. New York: Springer Publishing.

Fisher, PL., \& Wells, A. (2005). How effective are cognitive and behavioral treatments for obsessive-compulsive disorder? A clinical significance analysis. Behaviour Research and Therapy, 43, 1543-1558.

Gillihan S. J., Williams. M. T., Malcoun, E., Yadin, E. \& Foa, E.B (2012) Common pitfalls in exposure and response prevention (EX/RP) for OCD. Journal of Obsessive-Compulsive and Related Disorders ,1, 251-257.

Grayson, J. B. (2003). Freedom from Obsesive - Compulsive Disorder. Tarcher Penguin.

Grayson, J. B. (2010). OCD and Intolerance of Uncertainty: Treatment Issues. Journal of Cognitive Psychotherapy: An International QuarterlyVolume 24, 1, 3 - 15

Hiss, H., Foa, E. B. \& Kozak, M.J. (1994). Relapse prevention program of treatment of obsessive-compulsive disorder. Journal of Consulting and Clinical Psychology, 62, 801-808.

Julien, D., O'Connor K.P. \& Aardema, F. (2006) Intrussive thoughts, obssesions and appraisals on obsessive-compulsive disorder: A critical review. Clinical Psychology Review 27, 366-383

Kozak, M. J., \& Foa, E. B. (1994). Obsessions, overvalued ideas, and delusions in obsessive compulsive disorder. Behaviour Research andTherapy, 21, 57-62.

Mancini,F., D'Olimbio, F., Del Genio, M., Didonna, F. \& Prunetti E (2002): Obsessions and compulsions and intolerance for uncertainty in a nonclinical sample. Journal of Anxiety Disorders, 16, 401-411.

Melli, G., Aardema, F. \& Moulding, R. (2015). Fear of self and unacceptable thoughts in obsessive-compulsive disorder. Clinical Psychology \& Psychotherapy.

NICE (2006). National Clinical Practice Guideline Number 31. Obsessive Compulsive Disorder: Core interventions in the treatment of Obsessive Compulsive Disorder and Body Dysmorphic Disorder.

Obsessive Compulsive Cognitions Working Group (1997). Cognitive assessment of obsessive-compulsive disorder. Behaviour Research and Therapy, 35, 667-681.

O'Connor, K. \& Robillard, S. (1995). Inference processes in obsessive-compulsive disorder: Some clinical observations. Behaviour Research and Therapy,33, 887-896.

O'Connor, K. \& Robillard S. (1999). A cognitive approach to the treatment of primary inferences in obsessive-compulsive disorder. Journal of Cognitive Psychotherapy: An International Quarterly, 13, 359-375.

O'Connor, K. (2002) Intrusions and Inferences in Obsessive Compulsive Disorder. Clinical Psychology and Psychotherapy,9, 38-46

O'Connor, K., Aardema F. \& Pélissier M-C (2005)Beyond Reasonable Doubt: Reasoning Processes in Obsessive-Compulsive Disorder and Related Disorders. John Witley and Sons Ltd. Inglaterra

O'Connor, K., Aardema, F., Bouthillier, D., Fournier, S.,Guay S., Robillard S.,Pélissier, M.C. \& Landry P. (2005) Evaluation of an Inference-Based Approach to Treating Obsessive-Compulsive DisorderCognitive Behaviour Therapy Vol 34, No 3, pp. 148-163

O'Connor, K. \& Aardema, F. (2012) Clinician's handbook for obsessive-compulsive disorder: inference-based therapy (First Edition)Nueva Jersey: John Wiley \& Sons.

Pence, S.L., Sulkowsky, M.L., Jordan, C. y Storch, E. A (2010). When exposure go wrong: Trouble shooting guidelines for managing difficult scenarios that arise in exposure based treatment for Obsessive-Compulsive Disorder. American Journal of Psychotherapy. 64 - 1. $39-53$. 
Purcell Lalonde, M., O'Connor, K., Aardema, F. \& Coelho, J.S., 2015. Food for Thought: Ego-dystonicity and Fear of Self in Eating Disorders. European eating disorders review: the Journal of the Eating Disorders Association, 23(3), pp. 179-184.

Purdon, C. \& Clark, D. (1999) Metacognition and obsessions. Clinical Psychology and Psychotherapy. 6, 102-110

Rachman, S.J. (1994). Pollution of the Mind. Behaviour Research and Therapy, 32, 311 - 314

Rachman, S.J. (1997). A cognitive theory of obsessions. Behaviour Research and Therapy, 35, 793-802.

Rachman, S.J. (1998). A cognitive theory of obsessions: Elaborations. Behaviour Research and Therapy, 36, 385-401.

Rachman, S.J. (2003). The treatment of obsessions. Oxford, UK: Oxford University Press.

Rachman, S. J.,Coughtrey, A., Shafran, R., \&Radomsky, A. (2015). Oxford Guide to the Treatment of Mental Contamination. Oxford, UK: Oxford University Press.

Salkovskis, P.M. (1985). Obsessional-compulsive problems: A cognitive-behavioural analysis. Behaviour Research and Therapy, 23, 571-583.

Salkovskis, P.M. (1989). Cognitive-behavioural factors and the persistence of intrusive thoughts in obsessional problems. Behaviour Research and Therapy,27, 677-682.

Salkovskis, P. M. (1999). Understanding and treating obsessive compulsive disorder. Behaviour Research and Therapy, 37, S29-S52.

Salkovskis P. M., Shafran R., Rachman, S. \& Freeston, M. H. (1999a). Multiple pathways to inflated responsibility beliefs in obsessional problems: possible origins and implications for therapy and research. Behaviour Research andTherapy, 37, 1055-1072.

Steketee, G.S. (1993) Treatment of obsessive compulsive disorder. New York, Guilford

Steketee, G., \& Shapiro, L.J. (1993). Obsessive compulsive disorder. In A. S. Bellack \& M. Hersen (Eds) Handbook of Behavior Therapy in the Psychiatric Setting (pp. 199-227). New York: Plenum.

Tolin, DF. Worhunsky, P. \& Maltby, N. (2006) Are 'obsessive' beliefs specific to OCD? A comparison across anxiety disorders. Behaviour Research and Therapy, 44, 469-480.

Van Niekerk, J (2009) Coping with Obsessive - Compulsive Dosirder: a step-by-step guide usign the latest CBT techniques. Oneworld Publications.

Van Niekerk, J; Brown, G; Aardema, F y O'Connor, K. (2014) Integration of Inference-BAsed Therapy and Cognitive-Behavioural Therapy for Obsessive-Compulsive Disorder - A case series. International Journal of Cognitive Therapy, 7, 67 - 82

Veale, D. (2002) Over-valued ideas: a conceptual analysis. Behaviour Research and Therapy, 40, 383-400.

Wells, A. (1997) Cognitive therapy of anxiety disorders. a practice manual and conceptual guide. Chichester, UK: John Wiley and Sons Ltd. 